\title{
Chronic kidney disease of unknown aetiology: the tip of the iceberg?
}

Buddhika T B Wijerathne ${ }^{1}$, Robert John Meier $^{2}$, Liyanarachchige S S Salgado ${ }^{3}$, Geeta K Rathnayake, Sarath S Kumara ${ }^{5}$, Suneth B Agampodi ${ }^{1}$

${ }^{1}$ Faculty of Medicine and Allied Sciences, Rajarata University, Sri Lanka

${ }^{2}$ Indiana University, Bloomington, USA

${ }^{3}$ Faculty of Medicine, University of Kelaniya, Ragama, Sri Lanka

${ }^{4}$ Bankstown-Lidcombe Hospital, Sydney, Australia

${ }^{5}$ Divisional Hospital Thammannawa, Anuradhapura, Sri Lanka

\section{To the editor:}

Chronic kidney disease of unknown origin (CKDu) is a leading public health concern in Sri Lanka. It is not attributable to any common risk factor, such as diabetes, hypertension or glomerular diseases[1]. Despite being in an advanced stage of chronic kidney diseas(CKD), a significant number of patients remain asymptomatic[2-4]. Thus, the primary care physician may encounter patients with an advanced stage of CKDu who are seeking medical care for other health concerns We wish to write on patients have encountered this phenomenon during a study performed for screening of healthy individuals from a $\mathrm{CKDu}$ endemic region in Sri Lanka. Healthy individuals without a history or symptoms of kidney disease, hypertension, diabetes and snake envenoming, were invited to participate in our study as healthy controls in our CKDu study. All healthy participants were screened to exclude CKD before recruited into the study. Out of 194 screened individuals, five patients (Table 1) fulfilled the criteria for diagnosing CKDu based on the definition used in the study conducted by the World Health Organisation (WHO) and the Ministry of Health, Sri Lanka [1].

All five patients had never been screened for CKD, despite three of them having had CKD affected family members. (Table 1) All patients were farmers and three of them were in their 40s. Designated patient T16 was detected to have high BP as well as a history of headaches for four years, and he was referred immediately to the nearest divisional hospital (Thammanawa Hospital). This patient reported that he had sought medical care for an upper respiratory tract infection from a general practitioner two weeks prior to participating in our screening program, but his BP was not measured at that time. His BP was found to be normal a year before during a primary care consultation for a respiratory condition. (Note: Because there was a lack of previous BP measurement records the cause for CKD in this patient could have been undiagnosed hypertension). The remaining four patients were referred to a tertiary care nephrology unit and repeat renal function tests done at the renal clinic over a 3-month time period confirmed a diagnosis of CKD. Importantly, none of the patients had reported clinical symptoms except for T16.

Despite having a comprehensive community based CKD awareness and a screening program in disease endemic regions, asymptomatic patients with severe renal impairment may not always be discovered. This is especially true for male farmers, who apparently are not willing to participate in screening programs, unless they are symptomatic. However, the majority of these patients frequently seek medical care for common diseases at the primary care level. Thus, primary care physicians practicing in CKDu high endemic regions should be more vigilant and should engage in active case detection through opportunistic screening with serum creatinine and urine albumin-to-creatinine ratio[5], even in the absence of common risk factors for CKD. Individuals showing abnormal results during screening will be required to be referred to the nearest CKD clinic in the relevant endemic region for nephrologist's opinion and further evaluation. In addition, we recommend that health education and
Keywords: Chronic kidney disease;, chronic kidney disease of unknown aetiology, primary care screening, Sri Lanka

Date of submission: 18.102018

Date of acceptance: 20.02.2019

https://orcid.org/0000-0002-5378-6065

DOI: http://doi.org/10.4038/cjms.v55i2.4941

\author{
Author responsible for correspondence: \\ Dr. Buddhika TB Wijerathne \\ Department of Community Medicine \\ Faculty of Medicine and Allied Sciences \\ Rajarata University of Sri Lanka, Saliyapura, \\ Sri Lanka, 50008 \\ Email: buddhikatbw@gmail.com
}


health promotion regarding CKD be strengthened among public living in CKD endemic regions.

The authors declare no conflicts of interest

\section{Acknowledgement: None}

\section{References}

1 Jayatilake N, Mendis S, Maheepala P, et al. Chronic kidney disease of uncertain aetiology: prevalence and causative factors in a developing country. BMC Nephrol 2013;14:180. doi:10.1186/1471-2369-14-180

2 Wijetunge S, Ratnatunga NVI, Abeysekera TDJ, et al. Endemic chronic kidney disease of unknown etiology in Sri Lanka: Correlation of pathology with clinical stages. Indian J Nephrol 2015;25:274. doi:10.4103/0971-4065.145095

3 Wijetunge S, Ratnatunga NVI, Abeysekera DTDJ, et al. Retrospective analysis of renal histology in asymptomatic patients with probable chronic kidney disease of unknown aetiology in Sri Lanka. Ceylon Med J 2013;58:142-7. doi: $10.4038 / \mathrm{cmj} . v 58 \mathrm{i} 4.6304$

4 Jayasumana C, Orantes C, Herrera R, et al. Chronic interstitial nephritis in agricultural communities: a worldwide epidemic with social, occupational and environmental determinants. Nephrol Dial Transplant 2016;NDTAdvanc:gfw346. doi:10.1093/ndt/gfw346

5 Moyer VA, U.S. Preventive Services Task Force. Screening for chronic kidney disease: U.S. Preventive Services Task Force recommendation statement. Ann Intern Med 2012;157:567-70. doi:10.7326/0003-4819-157-8-201210160-00533 


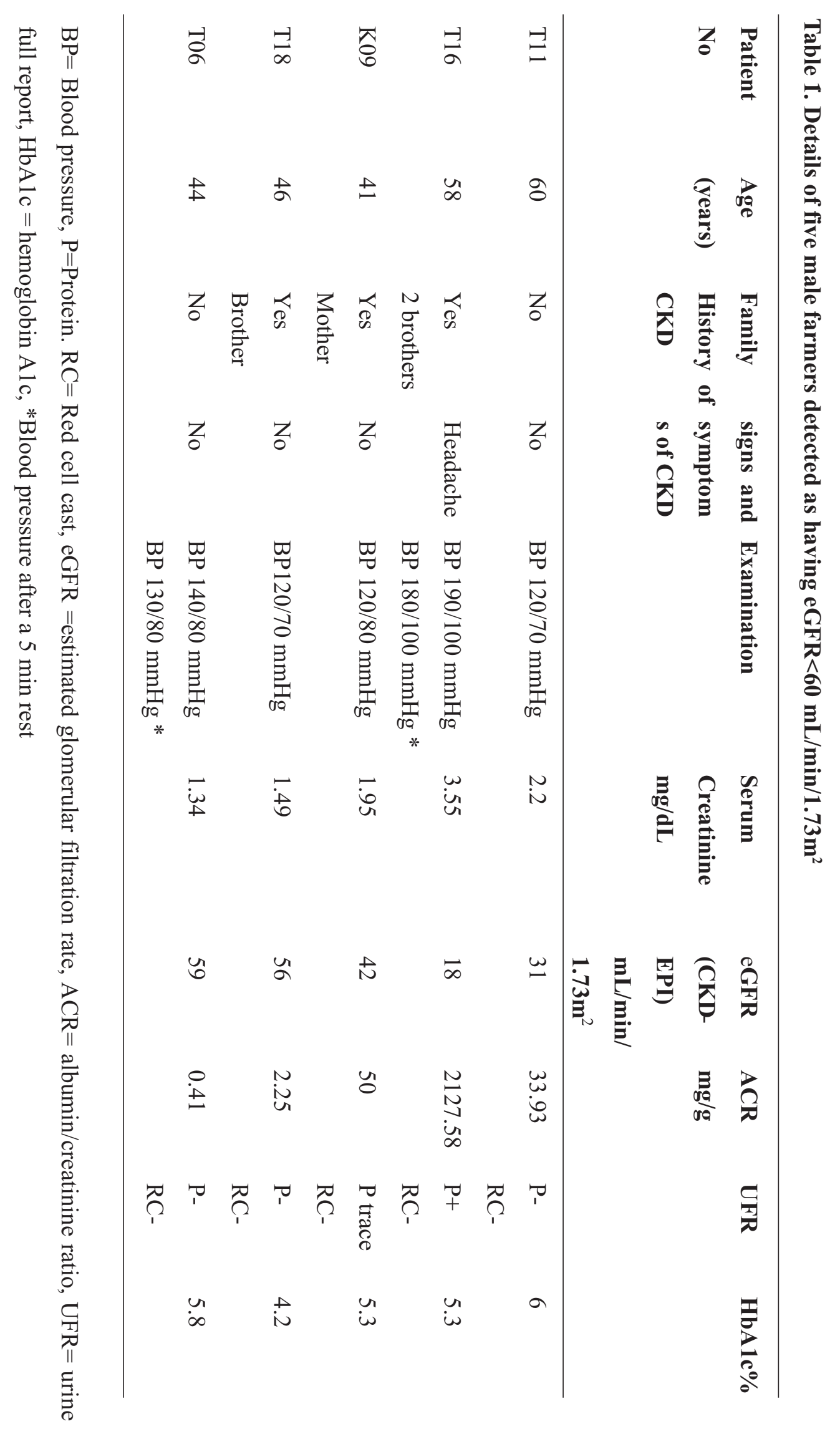

